\title{
An atomistic model for localized deformation
}

\author{
L. Teneketzis Tenek ${ }^{\prime}$ and E.C. Aifantis ${ }^{1,2^{*}}$ \\ 'Laboratory of Mechanics and Materials, Polytechnic School, 'A ristotle University of Thessaloniki, \\ GR-54124, Thessaloniki, Greece \\ ${ }^{2}$ Center for Mechanics of Materials and Instabilities, Michigan Technological University, \\ Houghton, MI 49931, USA
}

\begin{abstract}
We consider the discretization of a continuum in a number of atoms that exert attraction and repulsive forces. We develop a line finite element (similar to a truss finite element) that includes atoms at its nodes. The interaction of atoms is considered as in a finite element sense. Then, we postulate the elemental matrices such as the stiffness matrix, and the damping and mass matrices. Rigid body motion of the element is considered. A dynamic problem in time is formulated and advanced using a finite difference scheme. We apply the formulation to shear of a specimen and using a bilinear material law we realize the forming shear band. The force-displacement curve is plotted. The model is open to any number of atoms and can include numerically any material law. It is found that the shear band, a material instability, has chaotic characteristics.
\end{abstract}

\section{INTRODUCTION}

The present work is inspired and based on a dynamic approach known as Molecular Dynamics method $/ 1,2 /$ where a material or structure is assumed as an assembly of atoms or molecules and its motion is based on Newtonian deterministic dynamics given their initial positions and velocities. In this respect, the atoms experience Newtonian forces. The assumption is that each atom interacts with its nearest neighbours that, as in some works, lie within a specified cut-off radius. The motion in time, for their motion is dynamic in nature, can be obtained by a variety of finite difference techniques which update their positions and velocities. A popular algorithm is the Verlet algorithm $/ 3 /$. The energetics and dynamics of the atoms are obtained from prescribed phenomenological interatomic potentials /4/. During simulation, values of observable such as pressure, temperature and thermodynamics response functions are also obtained.

\footnotetext{
- Corresponding author
} 
Failure in materials involves localization of deformation, often involving small zones of highly strained material that develop abruptly while the remainder of the structure may not experience any additional straining. An example is the formation of shear bands in metals. Experiments show that localization phenomena cause a sharp decrease of the load-carrying capacity - a phenomenon commonly named strain softening.

In the present work we replace the continuum with an assembly of atoms and we consider a finite element that resembles a truss comprising two atoms (two nodes) on its edges. The two atoms with their respective masses are connected like a truss (or spring), which has an elastic stiffness and a damper. We formulate the mass matrix, the elastic stiffness and the damping matrix. We then assemble all the elements and atoms. All quantities are derived explicitly. A very significant part of the motion is the rigid body motion of the atoms and this is taken into consideration. The rigid body elemental motion is required for the formation of the mass matrix. The problem is dynamic in nature and the classical equation of dynamics is used to move the atoms in time. We then study the shearing of a small specimen investigating the phenomenon of shear band. Naturally, the model and associated computer program is open to any number of atoms only constrained by the available computer memory and speed.

Finite element techniques have been used in molecular dynamics simulations /5/. Atomistic computations involving shear banding are reported in $/ 6 /$. Transverse shearing of single and bimaterial systems using molecular dynamics has been studied in $17 \%$. Associated work in plastic flow and fracture including shear band can be seen in 18/. Plasticity and shear banding using a gradient-dependent theory can be found in 19/. The basic concepts of the truss finite element we developed in this study were taken from reference /10/. Deformation controlled uniaxial tension tests and modelling using beam elements have been reported in /11/.

\section{COMPUTATIONAL MODEL}

Figure 1 shows a specimen clamped at its bottom edge. The continuum is then replaced by a number of atoms and forces exerted between them. Every pair of atoms belongs to a line finite element as shown in the figure. We will refer upon this element as a truss finite element. Some basic notions of its mechanical behaviour are taken from reference $/ 10 /$. Here we will state some other important matrices such as the mass matrix. The finite element is shown in Figure 2. We observe the nodes (the atoms) the coordinate systems, the displacement fields and the one straining mode along its axis. The truss element with atoms at its nodes has a total of six Cartesian degrees of freedom representing the three displacement components at each of its nodes. We then assign to the truss finite element five rigid body modes and a straining mode and we ensure that rigid body motion does not enter in the expression of the elastic stiffness matrix. We then develop its elastic, damping and mass matrices, all derived explicitly. 


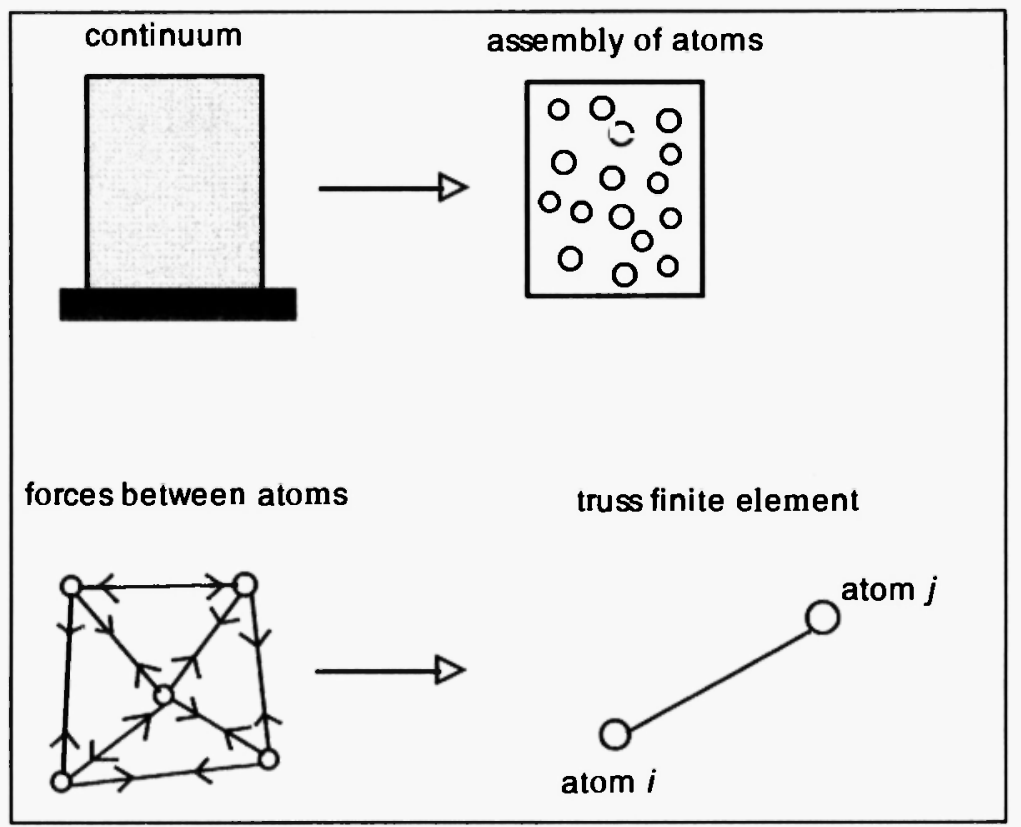

Fig. 1: Continuum modelization; forces between atoms

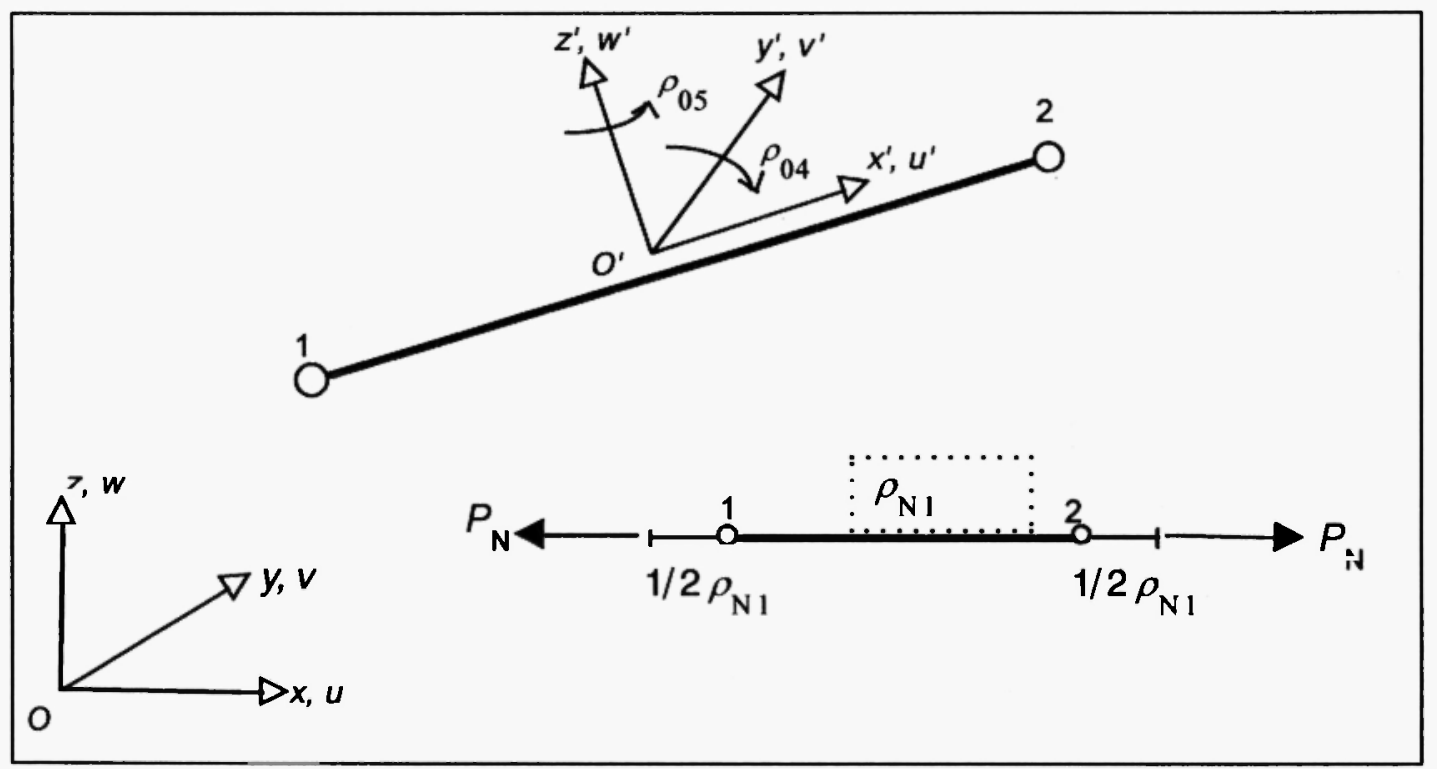

Fig. 2: Truss finite element connecting atoms

The truss element is space has two nodes and six global unknowns, namely three translations in node 1 and three translations at node 2 (see Fig. 2). With respect to a global coordinate $x y z$, its nodal degrees of freedom are grouped in the vector 


$$
\underset{(6 x 1)}{\rho}=\left\{\begin{array}{llllll}
u_{1} & v_{1} & w_{1} & u_{2} & v_{2} & w_{2}
\end{array}\right\}
$$

With respect to a local coordinate system $x^{\prime} y^{\prime} z^{\prime}$ placed at the element's center as Fig.2 indicates, the nodal unknowns are

$$
\underset{(6 \times 1)}{\rho^{\prime}}=\left\{\begin{array}{llllll}
u_{1}^{\prime} & v_{1}^{\prime} & w_{1}^{\prime} & u_{2}^{\prime} & v_{2}^{\prime} & w_{2}^{\prime}
\end{array}\right\}
$$

The truss element can move as a rigid body in space in five possible ways. There is also a natural straining mode, describing only the axial straining of the member and simulating the force exerted between atoms. The connection between the natural degree of freedom and the local and global Cartesian degrees of freedom is provided solely by matrix expressions. The natural rigid body modes include three translations $\rho_{01}, \rho_{02}, \rho_{03}$, along axes $x^{\prime}, y^{\prime}, z^{\prime}$, respectively, and two rotations $\rho_{04}, \rho_{05}$ with respect to axes $y^{\prime}$, and $z^{\prime}$, respectively (see Fig. 2). The three rigid-body translations are taken as the average of the corresponding translational nodal degrees of freedom. We thus postulate an equation expressing the relation between natural rigid-body modes and local Cartesian degrees of freedom. It reads

$$
\rho_{0 R}=\overline{\mathbf{a}}_{0 R} \rho^{\prime}
$$

or using matrix notation

$$
\left[\begin{array}{l}
\rho_{01} \\
\rho_{02} \\
\rho_{03} \\
\rho_{04} \\
\rho_{05}
\end{array}\right]=\left[\begin{array}{cccccc}
\frac{1}{2} & \cdot & \cdot & \frac{1}{2} & \cdot & \cdot \\
\cdot & \frac{1}{2} & \cdot & \cdot & \frac{1}{2} & \cdot \\
\cdot & \cdot & \frac{1}{2} & \cdot & \cdot & \frac{1}{2} \\
\cdot & \cdot & \frac{1}{l} & \cdot & \cdot & -\frac{1}{l} \\
\cdot & -\frac{1}{l} & \cdot & \cdot & \frac{1}{l} & \cdot
\end{array}\right]\left[\begin{array}{l}
u_{1} \\
v_{1} \\
w_{1} \\
u_{2} \\
v_{2} \\
w_{2}
\end{array}\right] .
$$

We place the natural coordinate axis $\alpha$ along the truss and the local cartesian coordinate $x^{\prime} y^{\prime} z^{\prime}$ at the member centre. We shall adopt a non-dimensional coordinate $\zeta$ defined as

$$
\zeta=\frac{2 x}{l}, \quad-1 \leq \zeta<1
$$

The natural straining mode is a unit extension. Half is assigned to the left edge and half to the right. Then, the axial displacement varies along the member axis as 


$$
u_{\alpha}=\frac{1}{2} \varsigma \rho_{N 1 .} .
$$

The strain caused by this displacement is

$$
\gamma_{t}=\frac{\partial u_{\alpha}}{\partial x}=\frac{\partial u_{\alpha}}{\partial \zeta} \frac{\partial \zeta}{\partial x}=\frac{1}{l} \hat{\nu}_{N 1}=\hat{u}_{N} \hat{\gamma}_{N 1}, \quad \hat{u}_{N}=t^{-1} \text {. }
$$

The natural straining mode is related to the vector of local Cartesian displacements via

$$
\underset{(1 \times 1)}{\rho_{N}}=\overline{\mathbf{a}}_{N} \underset{(1 \times 6)(6 x 1)}{\rho} .
$$

Since axis $x^{\prime}$ is parallel to the natural axis to the natural axis $\alpha$

$$
\rho_{N}=u_{2}^{\prime}-u_{1}^{\prime}
$$

Therefore

$$
\bar{a}_{N}=\left[\begin{array}{llllll}
-1 & . & & 1 & . & .
\end{array}\right.
$$

The complete matrix connection between the natural and local Cartesian degrees of freedom is provided by the matrix

$$
\bar{a}=\left[\begin{array}{c}
\bar{a}_{0} \\
\overline{\mathbf{a}}_{N}
\end{array}\right]=\left[\begin{array}{cccccc}
1 / 2 & . & . & 1 / 2 & . & . \\
\cdot & 1 / 2 & . & . & 1 / 2 & . \\
\cdot & . & 1 / 2 & . & . & 1 / 2 \\
. & . & 1 / l & . & . & -1 / l \\
. & -1 / l & . & . & 1 / l & . \\
\ldots . . & \ldots . . & \ldots \ldots & \ldots \ldots & \ldots . . & \ldots . . \\
-1 & . & . & 1 & . & .
\end{array}\right]
$$

The local Cartesian vector is connected to the global Cartesian vector via

$$
\rho^{\prime}=\mathbf{T}_{\mathbf{0 2}} \rho,
$$

where 


$$
\boldsymbol{T}_{02}=\left[\begin{array}{cc}
T_{0} & \cdot \\
\cdot & T_{0}
\end{array}\right] .
$$

Matrix $T_{0}$ includes a set of direction cosines between the local coordinate $x^{\prime} y^{\prime} z^{\prime}$ and the global coordinate $x y z$ as follows:

$$
T_{\partial}=\left[\begin{array}{lll}
c_{x^{\prime} x} & c_{x^{\prime} y} & c_{x^{\prime} z} \\
c_{y^{\prime} x} & c_{y^{\prime} y} & c_{y^{\prime} z} \\
c_{z^{\prime} x} & c_{z^{\prime} y} & c_{z^{\prime} z}
\end{array}\right] .
$$

On account of (12), (19) and (23) we postulate

$$
\gamma_{t}=\alpha_{N} \rho_{N}=\alpha_{N} \bar{a}_{N} \rho^{\prime}=\alpha_{N} \bar{a}_{N} T_{02} \rho \Rightarrow \rho_{N=} \bar{a}_{N} \boldsymbol{T}_{02} \rho
$$

From the energy expression we deduce we deduce the stiffness matrices of the truss finite element in the local and global Cartesian coordinates $\boldsymbol{k}$ ' and $\boldsymbol{k}$, respectively, as well as the initial global load due to temperature $\boldsymbol{J}$ as

$$
\begin{aligned}
& \underset{(6 \times 6)}{k^{\prime}}=\underset{(6 \times 1)(1 \times 1)(1 \times 6)}{\bar{a}_{N}^{t}} k_{N} \bar{a}_{N}=\bar{a}_{N}^{t}\left(\frac{A E}{i}\right) \bar{a}_{N},
\end{aligned}
$$

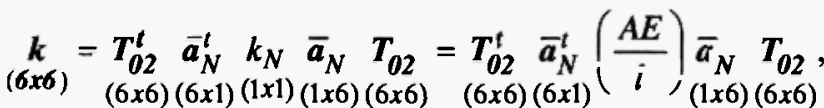

$$
\begin{aligned}
& \underset{(6 x i)}{J}=T_{02}^{t} \quad \bar{a}_{N}^{t} \quad J_{N}=T_{02}^{t} \quad \bar{a}_{N}^{t}\left[\alpha_{t}(E A) T\right] \\
& \text { (6xi) } \quad(6 \times 6)(6 \times 1)(1 \times 1) \quad(6 \times 6)(6 \times 1)
\end{aligned}
$$

With respect to the global coordinate system, the stiffness matrix of the truss element explicitly reads

$$
\underset{(6 x 6)}{\boldsymbol{k}}=\frac{A E}{l}\left[\begin{array}{cccccc}
c_{x^{\prime} x}^{2} & c_{x^{\prime} x} c_{x^{\prime} y} & c_{x^{\prime} x} c_{x^{\prime} z} & -c_{x^{\prime} x}^{2} & -c_{x^{\prime} x} c_{x^{\prime} y} & -c_{x^{\prime} x} c_{x^{\prime} z} \\
& c_{x^{\prime} y}^{2} & c_{x^{\prime} y} c_{x^{\prime} z} & -c_{x^{\prime} x} c_{x^{\prime} y} & -c_{x^{\prime} y}^{2} & -c_{x^{\prime} y} c_{x^{\prime} z} \\
& & c_{x^{\prime} z}^{2} & -c_{x^{\prime} x} c_{x^{\prime} z} & -c_{x^{\prime} y} c_{x^{\prime} z} & -c_{x^{\prime} z}^{2} \\
& & & c_{x^{\prime} x}^{2} & c_{x^{\prime} x} c_{x^{\prime} y} & c_{x^{\prime} x} c_{x^{\prime} z} \\
& & & & c_{x^{\prime} y}^{2} & c_{x^{\prime} y} c_{x^{\prime} z} \\
& & & & & c_{x^{\prime} z}^{2}
\end{array}\right]
$$

In order to derive the mass matrix for the truss finite element, we shall need the matrix connection of the Cartesian displacement field to the natural rigid body and straining modes. This connection is expressed as 


$$
\underset{(3 x 1)}{u}=\underset{(3 \times 6)}{\omega} \underset{(6 x 1)}{\rho_{0, N}}=\underset{(3 \times 6)}{\omega}\left[\begin{array}{c}
\rho_{0} \\
(5 x 1) \\
\rho_{N} \\
(1 \times 1)
\end{array}\right]
$$

Analytically, this matrix relation reads

$$
\left[\begin{array}{l}
u \\
v \\
w
\end{array}\right]=\left[\begin{array}{cccccc}
1 & \cdot & \cdot & \cdot & \cdot & 1 / 2 \zeta \\
\cdot & 1 & \cdot & \cdot & l / 2 \zeta & \cdot \\
\cdot & \cdot & 1 & -l / 2 \zeta & \cdot & \cdot
\end{array}\right]\left[\begin{array}{c}
\rho_{01} \\
\rho_{02} \\
\rho_{03} \\
\rho_{04} \\
\rho_{05} \\
\rho_{N 1}
\end{array}\right] .
$$

The global mass matrix is then given by $/ 9,10 /$

$$
M=\left[\bar{a} T_{02}\right]^{t} \int_{V} \rho \omega^{t} \omega d V\left[\bar{a} T_{02}\right]
$$

\section{NUMERICAL EXAMPLE}

We consider a shear specimen shown in Figure 1. It is clamped at its bottom boundary. We replace the continuum with an assembly of atoms as in a triangular lattice. We consider a relatively simple computational model. We apply a constant displacement rate at the top boundary during the time deformation. The specimen size is $2 \times 2 \mathrm{~cm}$. The Young modulus is taken as $E=1 \mathrm{Gpa}$. We run the dynamic problem over 200 time steps with a time step equal to $d t=0.00001$ seconds. At the right and left boundaries we impose periodic boundary conditions. A bilinear material law shown in Fig. 3 is adopted. During each time step the applied forces are computed using this diagram and imposed on the specimen. Figure 4 shows the shear band obtained. Figure 5 plots the average force-displacement for bin 1 that is the left boundary. Figure 6 displays the attractor of displacement versus velocity for an atom in the middle of our specimen. Figure 7 is a graph of the largest Lyapunov exponent of the horizontal acceleration of the atom for which the attractor eas plotted in Figure 6. Over evolution time, the Lyapunov exponent is positive an indication that the dynamic process has chaotic characteristics. Indeed, the shear band is a material instability and as such it may inherit chaotic characteristics. 


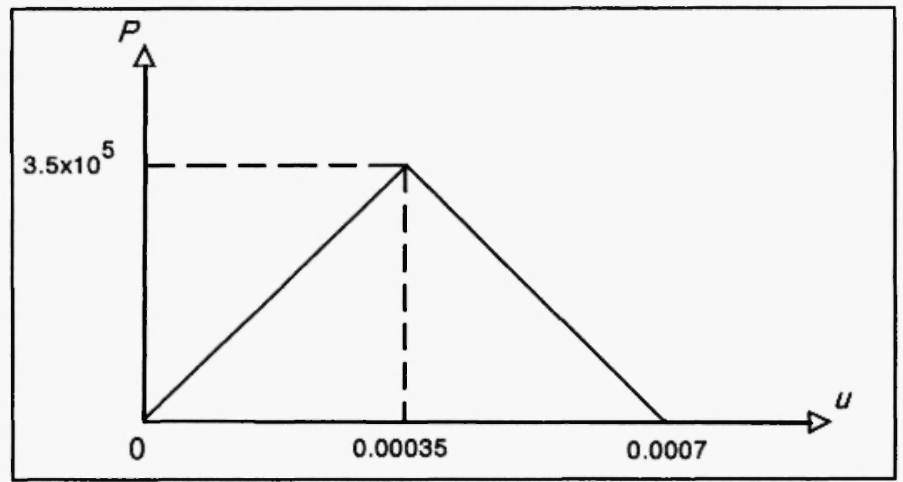

Fig. 3: Bilinear material law

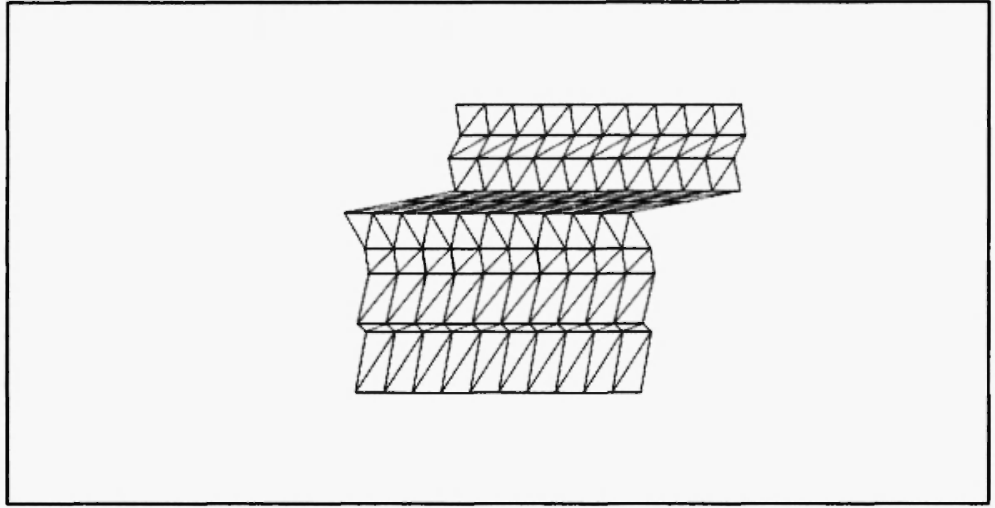

Fig. 4: Shear band

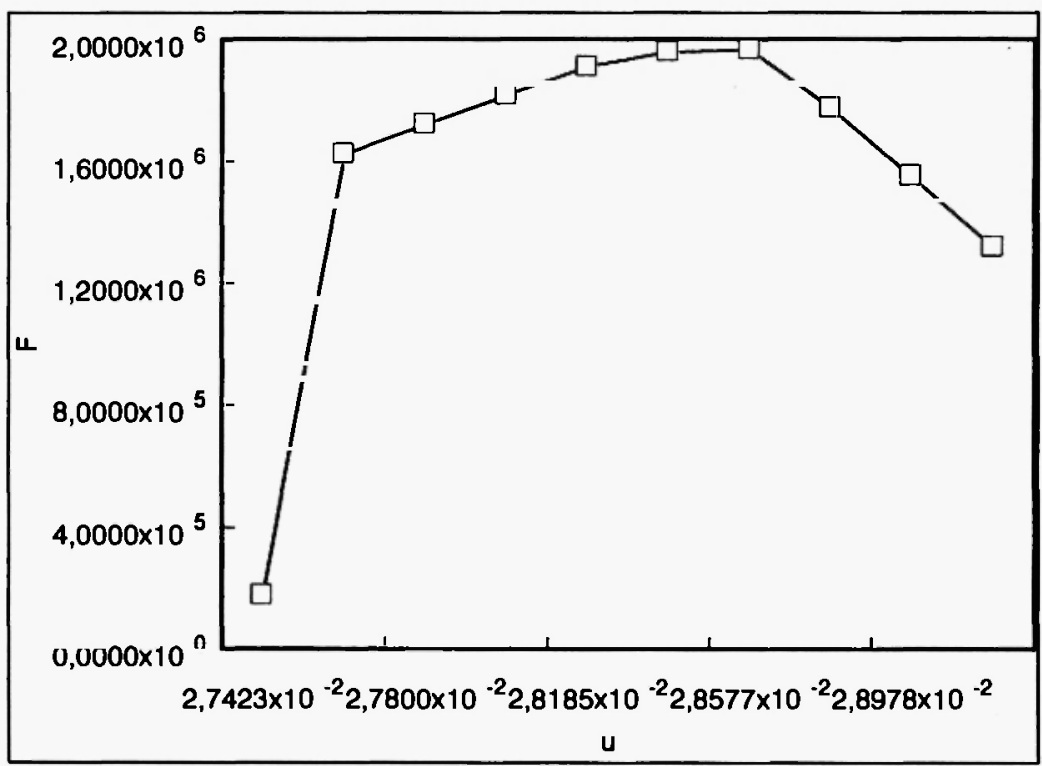

Fig. 5: Force versus displacement for left bin 


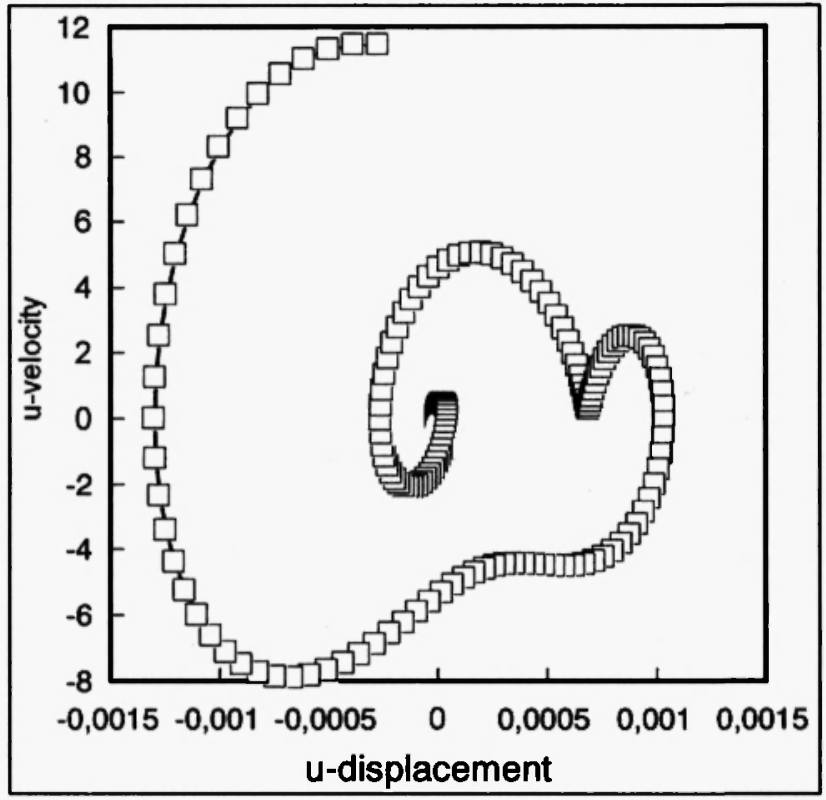

Fig. 6: Attractor for a specimen middle point

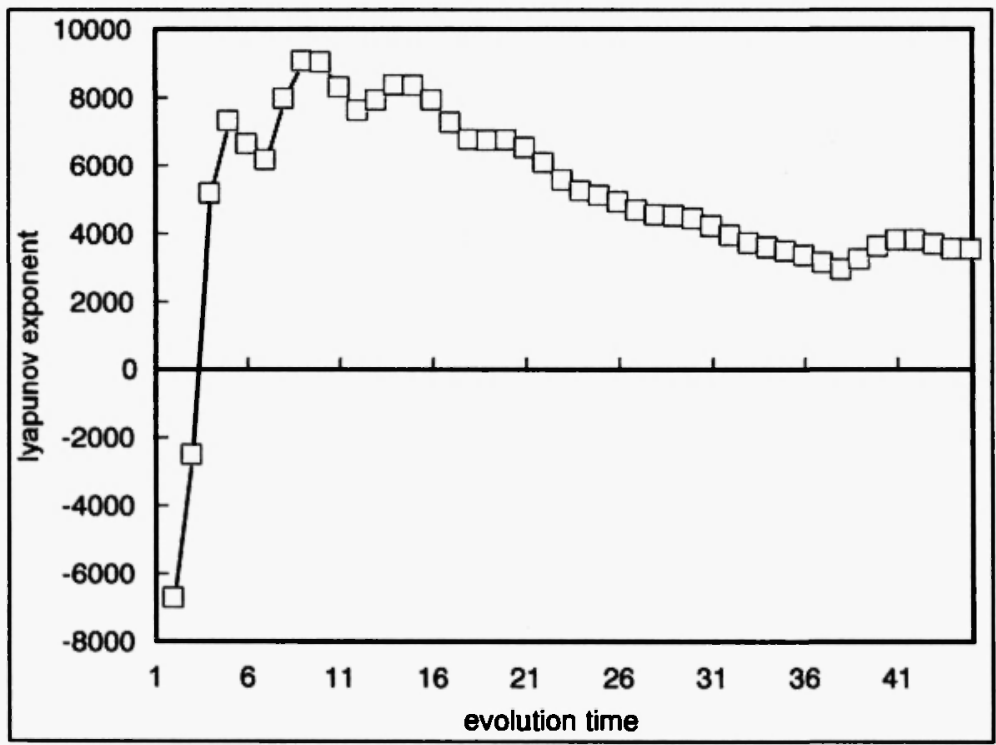

Fig. 7: The largest Lyapunov exponent

\section{REFERENCES}

1. J. M. Haile, Molecular Dynamics Simulation: Elementary Methods, Wiley, New York, 1992.

2. D. C. Rapaport, The Art of Molecular Dynamics Simulation, Cambridge University Press, Cambridge, UK, 1995. 
3. M. P. Allen and D. J. Tildesley, Computer Simulation of Liquids, Clarendon Press, Oxford, 1987.

4. H. Fafii-Tabar, Modelling the nano-scale phenomena in condensed matter physics via computer-based numerical simulations, Physics Reports, 325 (2000), pp. 239-310.

5. J. A. Smirnova, L. V. Zhigilei and B. J. Garrison, A combined molecular dynamics and finite element method technique applied to laser induced pressure wave propagation, Computer Physics Communications, 118 (1999), pp. 11-16.

6. M. F. Horstemeyer and M. I. Baskes, Atomistic Finite Deformation Simulations: A Discussion on Length Scale Effects in Relation to Mechanical Stresses, Journal of Engineering Materials and. Technology, Vol. 121, April 1999, pp. 114-119.

7. L. T. Tenek, M. I. Baskes and E. C. Aifantis, An Atomistic Approach to the Transverse Shearing of Single Aluminium and Aluminium-Nickel Bimaterial Systems, J. Mechanical Behaviour of Material, Vol. 14, N0 1, 2003, pp. 59-71.

8. E. C. Aifantis, Non-linearity, periodicity and patterning in plasticity and fracture, Int. J. Non-Linear Mechanics, Vol. 31, No 6, pp. 797-809.

9. H. M. Zbib and E. C. Aifantis, On the gradient-dependent theory of plasticity and shear banding, Acta Mechanica, 92 (1992), pp. 209-225.

10. J. H. Argyris and D. W. Scharpf, Large deflection analysis of prestressed networks, ASCE Journal of Structural Division, Vol. 98, No ST3, March 1972, pp. 633-654.

11. M. R. A Van Vliet and J. G. M. Van Mier, Experimental and Numerical Investigation of Size Scale Effects in Concrete Fracture, in Material Instabilities in Solids, Edited by Rene de Borst and Erik van der Giessen, 1998 John Wiley \& Sons Ltd.. 